\title{
Special relativity aces time trial
}

'Time dilation' predicted by Einste in confirmed by lithium ion experiment.

\section{Alexandra Witze}

19 September 2014

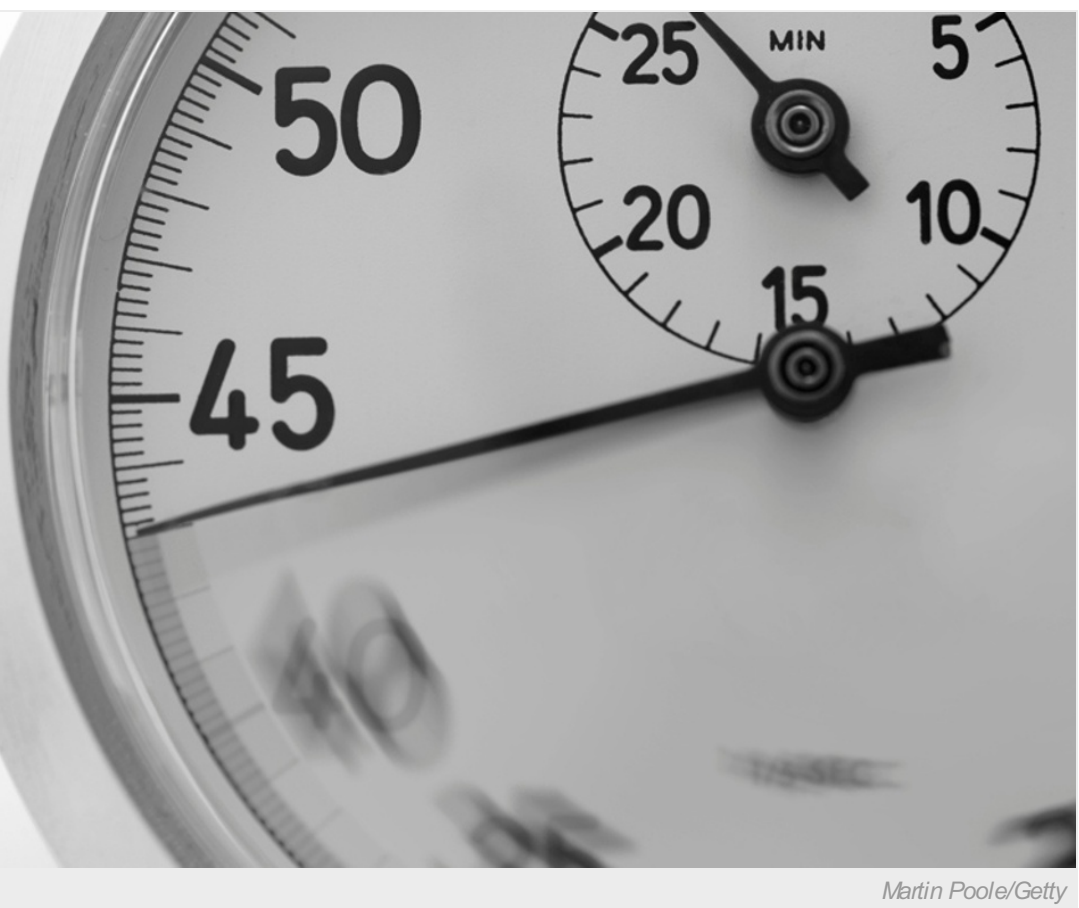

'Clocks' made of lithium ions helped researchers confirm the time-dilation effect predicted by Albert Einstein.

Physicists have verified a key prediction of Albert Einstein's special theory of relativity with unprecedented accuracy. Experiments at a particle accelerator in Germany confirm that time moves slower for a moving clock than for a stationary one.

The work is the most stringent test yet of this 'time-dilation' effect, which Einstein predicted. One of the consequences of this effect is that a person travelling in a high-speed rocket would age more slowly than people back on Earth.

Few scientists doubt that Einstein was right. But the mathematics describing the time-dilation effect are "fundamental to all physical theories", says Thomas Udem, a physicist at the Max Planck Institute for Quantum Optics in Garching, Germany, who was not involved in the research. "It is of utmost importance to verify it with the best possible accuracy."

The paper was published on 16 September in Physical Review Letters ${ }^{1}$. It is the culmination of 15 years of work by an international group of collaborators including Nobel laureate Theodor Hänsch, director of the Max Planck optics institute.

To test the time-dilation effect, physicists need to compare two clocks - one that is stationary and one that moves. To do this, the researchers used the Experimental Storage Ring, where high-speed particles are stored and studied at the GSI Helmholtz Centre for heavy-ion research in Darmstadt, Germany.

The scientists made the moving clock by accelerating lithium ions to one-third the speed of light. Then they measured a set of transitions within the lithium as electrons hopped between various energy levels. The frequency of the transitions served as the 'ticking' of the clock. Transitions within lithium ions that were not moving served as the stationary clock.

The researchers measured the time-dilation effect more precisely than in any previous study, including one published in 2007 by the same research group 2 . "It's nearly five times better than our old result, and 50 to 100 times better than any other method used by other people to measure relativistic time dilation," says co-author Gerald Gwinner, a physicist at the University of Manitoba in Winnipeg, Canada. 
Understanding time dilation has practical implications as well, he notes. Global Positioning System (GPS) satellites are essentially clocks in orbit, and GPS software has to account for tiny time shifts when analysing navigational information. The European Space Agency plans to test time dilation in space when it launches its Atomic Clock Ensemble in Space (ACES) experiment to the International Space Station in 2016.

The speed of fast-moving ions means that accelerator experiments can test time dilation more precisely than experiments in Earth orbit, says Matthew Mewes, a physicist at California Polytechnic State University in San Luis Obispo, who is not part of the team. "It's important to look wherever we can and push the technology whenever possible," he says.

But the research group is dismantling its longtime collaboration, as there is no larger accelerator they can go to for more powerful tests. "It's been many hours in basements, in shielded rooms with noisy equipment, and in the end you get one number," says Gwinner. "We've been exchanging a bunch of nostalgic e-mails."

Nature | doi:10.1038/nature.2014.15970

\section{References}

1. Botermann, B. et al. Phys. Rev. Lett. 113, 120405 (2014).

2. Reinhardt, S. Nature Phys. 3, 861-864 (2007). 